Cahiers $d u$ MONDE RUSSE

\section{Cahiers du monde russe}

Russie - Empire russe - Union soviétique et États indépendants

$52 / 4 \mid 2011$

Varia

\title{
Matthew E. Lenoe, The Kirov Murder and Soviet History
}

\author{
Gábor T. Rittersporn
}

\section{OpenEdition}

\section{Journals}

Édition électronique

URL : http://journals.openedition.org/monderusse/7622

DOI : $10.4000 /$ monderusse. 7622

ISSN : $1777-5388$

Éditeur

Éditions de l'EHESS

\section{Édition imprimée}

Date de publication : 20 décembre 2011

Pagination : 743-746

ISBN : 978-2-7132-2353-2

ISSN : $1252-6576$

Référence électronique

Gábor T. Rittersporn, "Matthew E. Lenoe, The Kirov Murder and Soviet History », Cahiers du monde russe [En ligne], 52/4 | 2011, mis en ligne le 30 novembre 2012, Consulté le 25 septembre 2020. URL http://journals.openedition.org/monderusse/7622 ; DOI : https://doi.org/10.4000/monderusse.7622

Ce document a été généré automatiquement le 25 septembre 2020

(c) École des hautes études en sciences sociales 


\title{
Matthew E. Lenoe, The Kirov Murder and Soviet History
}

\author{
Gábor T. Rittersporn
}

\section{RÉFÉRENCE}

Matthew E. LENOE, The Kirov Murder and Soviet History. New Haven, CT - Londres :

Yale University Press, 201, XxIV +832 p. (Annals of Communism)

1 La Commission Warren qui avait procédé aux investigations qui ont suivi l'assassinat de Kennedy avait alors publié un rapport de 888 pages. Le livre de Matthew E. Lenoe sur un autre crime historique est à peine moins volumineux. La Commission Warren était composée de sept membres. Plusieurs centaines de collaborateurs avaient amassé une volumineuse documentation, produisant une vingtaine de tomes de matériaux. Lenoe a travaillé seul et pour sa chance a dû évaluer une masse de sources moins volumineuse. Mais, pour sa malchance, il s'est heurté à une documentation complexe, contradictoire et lacunaire.

2 La Commission Warren avait à répondre à une seule question : l'assassin avait-il agi seul ? Lenoe aurait pu suivre son exemple et restreindre ses recherches à l'analyse d'un nombre limité de documents sur le problème qui intrigue beaucoup de monde depuis 1934 : peut-on découvrir la main de Stalin derrière le meurtre? Mais il a alourdi sa tâche en insérant l'attentat dans un contexte qui commence par la biographie de la cible, passe par les arcanes des intrigues au sein de l'État-parti, par l'évolution et les constantes des mentalités soviétiques, par la Grande Terreur de 1937-1938 et par la politique de réhabilitation des victimes à partir des années 1950 ainsi que par les diverses enquêtes de la police secrète et des sommets du parti qui n'ont pris fin qu'à la fin des années 1980. Même si la mort de Sergej M. Kirov est au cœur de l'ouvrage, les sujets dont l'auteur traite dépassent largement l'événement.

Lenoe a travaillé essentiellement à partir de sources inédites. Il a ainsi pu fouiller dans les archives du Comité central et, moyennant beaucoup d'habileté, il a réussi à mettre 
la main sur nombre de documents des organes de sécurité. L'historien n'a pas négligé la littérature qui était déjà à la disposition des chercheurs avant l'ouverture partielle des archives soviétiques et a même pris la peine de traduire du japonais le témoignage d'un participant clé des enquêtes policières des années 1930 qui est parvenu à fuir l'URSS. Le volume contient la traduction, tantôt in toto, tantôt in excerptio, de plus de cent vingt sources, presque toutes inconnues.

La nature des matériaux explique l'étendue de l'étude. Qu'il s'agisse d'affirmations de contemporains, de rapports ultrasecrets rédigés dans les années 1930 ou du résultat d'investigations ultérieures fondées sur ces derniers, la véracité ou l'exactitude de toutes les informations est suspecte. Lenoe déploie une connaissance profonde de l'univers soviétique et mille astuces de la philologie historique pour procéder à l'analyse critique des sources. Il repère des détails importants qui semblent sans signification au premier coup d'œil, il compare et recoupe des indications, peu importe si elles sont bien éloignées de l'affaire chronologiquement et proviennent de différents types de documents, il est prêt à écarter des éléments qui pourraient pourtant servir sa démonstration et, surtout, il n'ignore aucune hypothèse.

5 L'historien arrive à la conclusion que, contrairement à l'opinion de beaucoup de citoyens soviétiques et post-soviétiques et d'un certain nombre d'historiens russes et étrangers ${ }^{1}$, les matériaux ne permettent pas d'imputer à Stalin l'assassinat de son ami fidèle ni de découvrir un complot au sein de la police politique pour éliminer le secrétaire de la région de Leningrad et du Comité central. La conjecture selon laquelle le meurtrier voulait venger une liaison amoureuse entre sa femme et le haut notable se révèle fausse. Tout comme l' accusation de collusion avec des puissances étrangères.

Les rumeurs d'une rivalité entre Kirov et Stalin se révèlent ainsi sans fondement. Elles s'expliquent par un folklore particulier au sein du parti et dans le reste de la société, par un culte soviétique d'illustres défunts et par une tradition orale qui a fait son chemin jusqu'à la littérature savante de l'Occident. Fait intéressant: une bonne partie des spéculations concernant l'implication de Stalin dans ce crime et l'opposition de Kirov à son maître furent basées sur les aveux publiés par des accusés des grands procès de 1936-1938. Les gens qui diffusaient des histoires imaginaires et ceux qui les ont reprises à leur compte savaient bien comment on avait arraché de telles confessions. Parmi ceux-ci, certains en avaient fait l'expérience eux-mêmes. Mais peu importait la vraisemblance des informations à partir du moment où il s'agissait de se démarquer du projet soviétique ou de justifier son engagement pour le régime en tentant de le séparer des méfaits du dictateur.

7 Une partie des investigations que le parti a reprises à partir de 1953 furent destinées à prouver que les successeurs de Stalin n'étaient pas responsables de la Terreur. D'autres enquêtes étaient censées attester en fonction des rapports de force au sein du Comité central qu'un groupe de hauts dignitaires était coupable. Certains dirigeants voulaient disculper Stalin alors que leurs collègues avaient tout intérêt à le mettre en cause, ne serait-ce que par vagues insinuations. Les discours prononcés à des congrès du parti et répercutés au sein de l'organisation ou diffusés à l'intention du public portent l'empreinte de ces tiraillements. Il en va de même des matériaux publiés jusqu'à la fin des années 1980, même s'ils étaient des documents authentiques. Les citoyens et les chercheurs étaient libres de faire le tri pour démontrer la pertinence de n'importe quelle hypothèse. 
8 La situation est rendue encore plus complexe par les témoignages publiés et par ceux qui circulaient de bouche à oreille. Les informations de presque tous les témoins étaient de seconde ou troisième main, à moins qu'elles ne viennent de plus loin encore. La tradition orale a contaminé même les souvenirs de quelques témoins directs que la police et diverses commissions du parti ont interrogés dans les années 1950 et plus tard alors que les récits des autres sont documentables. Autant d'obstacles que Lenoe a surmontés. Mais autant d'éléments pour une meilleure connaissance du monde intérieur des Soviétiques.

9 L'historien a remué ciel et terre pour faire ressortir de l'obscurité l'assassin, Leonid V. Nikolaev. S'il se trouve que le peu d'informations dont on disposait à son sujet contenaient quelques détails plus ou moins fiables, le portrait dressé par Lenoe est unique. Âgé de trente ans au moment du crime, dont il a passé la moitié comme activiste du Komsomol et cadre subalterne du parti, Nikolaev fut longtemps un inconditionnel du régime. Il étudiait assidûment les doctrines canoniques, a travaillé un temps comme propagandiste et a donné à son premier fils le prénom «Marx ». Il était querelleux et mégalomane, conduisant ses collègues et ses supérieurs à tout faire pour se débarrasser de lui. Une occasion rêvée est arrivée quelques mois avant qu'il ne tue Kirov. Nikolaev a refusé une mutation et ses camarades ont saisi ce prétexte pour l'éloigner de l'appareil et l'exclure du parti. Commence alors pour lui une période de quête d'une réhabilitation marquée par des pétitions infructueuses adressées à Stalin et à sa future victime. Elle s'est achevée par l'expression d'une véritable rage contre les dirigeants.

10 Sur la foi des aveux que sa femme a faits après son arrestation, Nikolaev commençait à questionner le bien-fondé de la politique du parti après avoir fait des missions à la campagne où il avait été témoin de l'extorsion de la récolte des paysans. Selon elle, Nikolaev s'était mis à parler de l'exploitation des travailleurs et à critiquer les sommes exorbitantes investies dans l'armée. On retrouve ce genre de mécontentement dans les écrits intimes de nombre de fidèles désorienté par le régime ainsi que dans des tracts disséminés à l'époque dont l'authenticité ne fait aucun doute. Quoi qu'il en soit, quelques mois avant son coup de feu fatal, Nikolaev rédigeait des notes de plus en plus radicales dans son journal dont Lenoe a consulté de larges extraits et commençait à appeler Stalin le "Tsar de l'industrie et de la guerre », apostrophe qui apparaît aussi dans l'ébauche d'une missive qu'il voulait envoyer au Comité central. Ses griefs personnels se mêlaient à l'idée que la révolution n'avait rien changé vers le mieux et à la conviction qu'il serait impossible de construire le communisme en URSS, certitude qui fait surface dans les journaux de nombre d'autres déçus. Dans diverses versions d'un testament politique, il parlait de l'attentat qu'il allait commettre, se désignait comme soldat intrépide de la révolution et se comparait aux terroristes révolutionnaires du XIX ${ }^{e}$ siècle.

Nikolaev fut jugé et condamné à mort avec treize autres personnes. C'étaient en partie des collègues des années 1920 dont un certain nombre avait sympathisé avec l'opposition de gauche ou milité dans ses rangs. La plupart de ces gens rejetaient l'accusation de conspiration contre le régime. Il en allait de même pour Grigorii E. Zinov'ev, ancien homme fort de l'opposition et de Leningrad que Stalin a impliqué dans cette affaire après deux semaines d'hésitation. Le dictateur n'avait pas abandonné l'idée d'exploiter l'attentat et des prétendues conspirations pour régler de vieux comptes. Mais il est douteux qu'il ait envisagé une terreur à grande échelle au départ. 
On sait depuis quelques années que l'assassinat de Kirov n'a pas provoqué la vaste opération de purge qui fut lancée en 1937 que les contemporains lui ont attribuée des années plus tard. Des historiens leur ont emboîté le pas. Pourtant, pour ne citer qu'un exemple, le nombre des personnes que la police secrète a exécutées et celui des prisonniers prétendument politiques a baissé chaque année entre 1934 et 1937.

Même s'il avait su combien de malheureux furent accusés de comploter contre l'éminent défunt et son chef, Pascal se serait sans doute moqué de ceux qui ont prétendu que, pareillement à la longueur du nez de Cléopâtre, l'attentat a changé la face de la terre. Les tenants et les aboutissants de l'histoire en général et celle de l'Union soviétique en particulier sont plus complexes. Au fond, Stalin n'avait pas besoin de justifier sa politique par un meurtre politique susceptible de donner de bonnes idées à quelques-uns de ses sujets. Parallèlement au deuil tapageur de Kirov et à la glorification de sa mémoire, l'opinion que Nikolaev aurait plutôt dû tuer Stalin faisait son chemin. Elle fut souvent citée dans les rapports de la police et est apparue dans les écrits intimes. Stalin n'a déployé aucun effort pour légitimer devant le public la terreur déchaînée qu'il a déclenchée en 1937.

Toute une industrie produit depuis bientôt un demi-siècle d'innombrable articles et livres pour réfuter les conclusions de la Commission Warren. Les théories du complot fournissent des explications plus simples et plus aisées à digérer que les études multidimensionnelles qui supposent un certain travail intellectuel de la part des chercheurs et des lecteurs. Il se peut que les détracteurs de Lenoe aiguisent déjà leurs plumes. Ils auront quelques fils à retordre. Mais si la démonstration rigoureuse de l'historien ne facilitera pas leurs labeurs, la masse de matériaux qu'il a réunie fera leur bonheur. Espérons que les collègues qui cherchent au-delà des spéculations simplistes fassent bon usage des trouvailles de cet ouvrage impressionnant.

\section{NOTES}

1. Parmi les ouvrages qui ont fait longtemps autorité voir, en particulier, Boris Nikolaevsky, Power and the Soviet Elite, New York, 1965; Roy Medvedev, Le Stalinisme, P., 1972; id., Staline et le stalinisme, P., 1979 ; Anton Antonov-Ovseenko, In Stalin's Time: Portrait of a Tyranny, New York, 1981; Robert Conquest, The Great Terror, Londres, 1968; id., Stalin and the Kirov Murder, New York, 1989. 